\title{
Der Sandlöß in der Umgebung von Bergen Krs. Celle - Verbreitung, Zusammensetzung und Entstehung -
}

\author{
Hans DieTrich LANG *)
}

\begin{abstract}
Sandy loess, spatial distribution, thickness, stratification, bioturbation, cryoturbation, size distribution, paleorelief, winds, paleocurrents, provenance
\end{abstract}

Lower Saxony, Northwestern German Plain, TK 3125, 3126, 3225, 3226

\begin{abstract}
Kurzfassung: Das Sandlößgebiet um Bergen Krs. Celle erstreckt sich über $15 \mathrm{~km}$ in WNW-ESE-Richtung und etwa $12 \mathrm{~km}$ in NNE-SSW-Richtung, von der Falken-BergEndmoräne im W bis an das Tal der Örtze im E. Der Sandlöß erreicht eine Mächtigkeit von ca. 3,1 m. Sie ist am größten im äußersten NW des Verbreitungsgebietes.
\end{abstract}

Ein Streifen mit über $2 \mathrm{~m}$ mächtigem Sandlöß begleitet den $\mathrm{N}$-Rand des Verbreitungsgebietes. Nach S hin nimmt die Mächtigkeit ab.

Die obersten $0,7-0,9 \mathrm{~m}$ des Sandlösses sind ungeschichtet und weisen vielfach Wühlspuren auf. Ist er weniger mächtig als 0,7-0,9 m, enthält er einzelne Geschiebe bzw. Gerölle. Geht die Mächtigkeit darüber hinaus, ist der untere Teil sandstreifig, der obere Teil frei von Geröllen bzw. Geschieben. Je geringer die Mächtigkeit, um so geringer ist der Anteil an Feinmaterial und umso höher der Sandanteil.

Der Sandlöß war ursprünglich durchweg geschichtet. Bioturbation und Kryoturbation haben ihn bis zu etwa 0,7 bis $0,9 \mathrm{~m}$ Tiefe entschichtet und, wenn die Mächtigkeit nicht über dieses Maß hinausging, mit Material aus dem Liegenden vermischt.

Die Lage des Sandlößgebietes, seine Nachbarschaft zu Flugsandfeldern im N, die Verzahnung von Sandlöß mit Flugsand und die Mächtigkeitsverteilung sprechen für nordwestliche und nördliche bis nordöstliche Winde zur Zeit der Sandlöß-Entstehung.

[The Sandy Loess (Sandlöß) in the Bergen Area, Celle District - Distribution, Composition and Genesis -]

Abstract: The sandy loess area around Bergen in the District of Celle extends about $15 \mathrm{~km}$ in a WNW-ESE direction and approximately $12 \mathrm{~km}$ in a NNE-SSW direction, from the Falken-Berg endmoraine in the west down to the Örtze

*) Anschrift des Verfassers: Dr. H. D. LANG, Marienburger Weg 9, D - 3004 Isernhagen NB, b. Hannover. valley in the east. The maximum thickness of the sandy loess amounts to about $3.1 \mathrm{~m}$, being thickest in the farthest northwest of the distribution area.

A strip of sandy loess over $2 \mathrm{~m}$ thick runs along the northern boundary of the distribution area. The thickness decreases in a southern direction.

The uppermost $0.7-0.9 \mathrm{~m}$ of the sandy loess are unstratified, frequently showing signs of burrowing. When less than $0.7-0.9 \mathrm{~m}$, the sandy loess includes a few small boulders or pebbles. When the thickness exceeds this value the lower part is sandy and stratified and the upper part is free of pebbles or boulders. The thinner the layer of sandy loess the smaller the proportion of fine material in it and the greater the proportion of sand.

Originally the sandy loess was stratified throughout. Bioturbation and kryoturbation have destroyed the stratification down to a depth of $0.7-0.9 \mathrm{~m}$. Where the thickness of the sandy loess was greater than this, material from the underlaying bed was mixed up with the sandy loess.

The position of the sandy loess area, its proximity to the eolian sand fields in the north, the interfingering of the sandy loess with eolian sand, and the thickness distribution suggest a prevailing northernly to northeasternly wind during time that the sandy loess was deposited.

\section{Einführung}

Das Sandlößgebiet um Bergen Krs. Celle erstreckt sich etwa über $15 \mathrm{~km}$ in nordwest-südöstlicher und etwa $12 \mathrm{~km}$ in nordost-südwestlicher Richtung. Seine heutige zerlappte Form ist das Ergebnis der jungen, großenteils holozänen Erosion. Die Grenzen auf Abb. 1 umfahren das Gebiet, in dem der Sandlöß heute eine Mindestmächtigkeit von $30 \mathrm{~cm}$ hat. Zerwühlung, Durchwurzelung und Bodenbearbeitung haben ihn bei geringerer Mächtigkeit so stark mit dem Substrat 
vermischt, daß er nicht mehr einwandfrei identifizierbar ist.

Das Bergener Sandlößgebiet erstreckt sich über die Blätter 3125 Bergen, 3126 Hermannsburg, 3225 Offen und 3226 Sülze der Geologischen Karte 1: 25000. Der Großteil liegt auf Blatt Bergen. Es wird, abgesehen von den Randbereichen des Verbreitungsgebietes mit geringmächtigem Sandlöß, intensiv als Ackerland genutzt. Lediglich die Teile, die innerhalb des Endmoränen-Hochgebietes um den Falken-Berg liegen und die zum militärischen Übungsgebiet gehören, sind von Wald bestanden.

Die erste Beschreibung des Sandlösses aus diesem Raum gab STOLLeR (1915a, b) in den Erläuterungen zu den Blättern 3126 Hermannsburg und 3226 Sülze der Geologischen Karte 1:25000. Besonders mit Fragen der Stratigraphie und Genese, auch des Bergener Sandlösses, beschäftigte sich VIERHUFF (1967) in einer Untersuchung der Niedersächsischen Sandlößvorkommen. Den Sandlöß auf den Blättern Offen (LANG 1983) und Bergen (LANG 1989) untersuchte der Autor im Rahmen einer geologischen Kartierung.

Die Ergebnisse des Autors beruhen auf etwa 500-600 Bohrungen mit einem Peilstangen-Bohrgerät auf $\mathrm{Bl}$. 3125 Bergen der geol. Karte 1:25000 und etwa 150 solcher Bohrungen auf Bl. 3225 Offen, auf Aufschlußaufnahmen, Aufgrabungen und Korngrößenanalysen von 6 durchgehenden Profilen mit insgesamt 84 Proben. Die Korngrößenanalysen führte Herr Dr. MatTiat, Nieders. Landesamt $f$. Bodenforschung in Hannover aus, wofür ihm und seinen Mitarbeitern an dieser Stelle nochmals gedankt sei.

Zur Frage der Böden bzw. der Bodenbildung auf Sandlöß wird in der vorliegenden Arbeit nicht näher Stellung genommen. Im Rahmen der geologischen Kartierung fielen, auch bedingt durch die Arbeitsmethode, keine wesentlichen Beobachtungen zur Ausbildung der Böden auf Sandlöß an, die über das hinausgehen, was an anderer Stelle bzw. von anderen Autoren über Böden in Sandlößgebieten bisher berichtet wurde (DEWERS 1931/32; WORTMANN 1942; ROESCHMANN 1963, 1968; KLAUSING in LANG 1990). Auf dem Sandlöß im Raum Bergen hat sich eine Parabraunerde gebildet, die stellenweise leicht podsoliert ist. Die lebhafte braunrote Bänderung im Unterboden weist auf vertikale Tondurchschlämmung und auf eine Tonverarmung des Oberbodens hin.

Die Arbeiten haben auch keine neuen Aspekte zum Alter des Sandlösses ergeben. Es ist davon auszugehen, daß er im ausgehenden Hochglazial oder beginnenden Spätglazial abgelagert wurde (s. LANG 1974).

\section{Das Verbreitungsgebiet}

Der Sandlöß überzieht in einer maximal etwas mehr als $3 \mathrm{~m}$ mächtigen Decke das leicht gewellte Gebiet um Bergen mit Höhen zwischen 60 und $90 \mathrm{~m}$ üb. NN bis an das Örtze-Tal im E heran, überdeckt den östlichen Abhang des Endmoränen-Hochgebietes um den Falken-Berg und tritt im Becklinger Holz in einer flachen Schüssel innerhalb des Endmoränen-Hochgebietes in Höhen bis $130 \mathrm{~m}$ üb. NN auf (Abb. 1).

Innerhalb dieses Verbreitungsgebietes zeigt der Sandlöß nur wenig Anhängigkeit von den Oberflächenformen. Er überzieht in relativ gleichbleibender Mächtigkeit Senken und Rücken. So trägt der Mühlenberg-Zug, der aus der NE-Ecke des Blattes Bergen in südsüdwestlicher Richtung auf Bergen zu zieht, genau so eine Sandlößdecke wie seine um etwa $20 \mathrm{~m}$ niedrigere Umgebung, auch in sehr ähnlicher Mächtigkeit.

STOLLER (1915a, b) hat auf den Blättern Hermannsburg Nr. 3126 und Sülze Nr. 3226 der geologischen Karte 1:25000 „Flottsand” (früher üblicher Ausdruck für Sandlöß, genau so wie „Flottlehm”) mit einer Mächtigkeit bis zu 1,8 m dargestellt. Die Grenzen des Verbreitungsgebietes schließen zwanglos an die Grenzen des Sandlösses auf den Blättern Bergen Nr. 3125 und Offen Nr. 3225 an.

Das Verbreitungsgebiet des Sandlösses zeigt keine auffallende Abhängigkeit vom Substrat. Er tritt über allen älter als weichselzeitlichen Ablagerungen auf. Nach STOLLER (1916b) greift auf Blatt Sülze ,geschiebeführender Flottsand” auf "Talstufenreste des altdiluvialen Örtzetales" über, auch als ,älterer Talsand" bezeichnet. Nach den eigenen Untersuchungen schliessen sich Sandlöß und weichselzeitlicher fluviatiler Sand aus, da die Sedimentation des letzteren wohl länger angedauert hat als die des Sandlösses. Da die ,älteste Stufe des Talsandes” nach STOLLER (1915b) deutlich gegenüber der jüngeren abgesetzt ist, dürfte ein höheres als weichselzeitliches bzw. sehr frühes weichselzeitliches Alter nicht auszuschließen bzw. wahrscheinlich sein.

\section{Mächtigkeit}

Nach VIERHUFF (1967) wird der Sandlöß im Raum Bergen maximal 3,2 m mächtig, nach Beobachtungen des Autors 3,08 m. VIERHUFF gibt die größte Mächtigkeit aus der Nähe von Beckedorf auf Blatt 3226 Sülze an, nicht weit von der Ostgrenze des Verbreitungsgebietes entfernt. Nach den eigenen Beobachtungen wird er im Gebiet etwa $500 \mathrm{~m}$ westlich Becklingen, also nahezu im NW-Zipfel, am mächtigsten (Abb. 1). 
Größere zusammenhängende Flächen mit mehr als $2 \mathrm{~m}$ mächtigem Sandlöß treten zwischen dem Großen Moor und dem Endmoränen-Hochgebiet um den Falken-Berg auf, dazu in der Umgebung von Becklingen und Wardböhmen, mit Ausläufern nach Hoope bei Bleckmar. Relativ groß ist die Mächtigkeit ferner im Raum Bleckmar-Nindorf-Hagen, nahe dem S-Ende des Großen Moores. Auch hier haben wir wieder, wenn auch kleinere, Flächen mit mehr als 2 mächtigem Sandlöß. Diese Flächen liegen sämtlich im äußersten $\mathrm{N}$ bis NW des Verbreitungsgebietes, und die $2 \mathrm{~m}$ - und 1,5 m-Mächtigkeitslinien des Sandlösses lassen deutlich einen Streifen mächtigen Sandlösses erkennen, der nicht weit vom N-Rand des Verbreitungsgebietes von WNW nach ESE zieht (s. Abb. 1).

STOLLER (1915a) gibt von Blatt 3126 Hermannsburg 1-2 m mächtigen ,gerölle- und geschiebefreien Flottsand" an, vom südlich anschließenden Blatt 3226 Sülze lediglich „Geschiebeflottsand” von selten mehr als 0,6-0,8 m Mächtigkeit. Im südlichen Randgebiet des Blattes 3125 Bergen und auf dem südlich anschließenden Blatt Offen (LANG 1983) beträgt die Mächtigkeit fast durchweg weniger als $1 \mathrm{~m}$.

Die Abb. 1 zeigt ferner, daß Flugsandflächen den NRand des Verbreitungsgebietes begleiten.

Gar nicht in dieses Bild paßt die von VIERHUFF (1967) angegebene Mächtigkeit von 3,2 m aus der Umgebung von Beckedorf. Eigene Beobachtungen liegen aus diesem Raum jedoch nicht vor.

Der Trend in der Mächtigkeitsabnahme von $\mathrm{N}$ bis NW nach S bis SE wird von den Oberflächenformen innerhalb des Verbreitungsgebietes nur wenig beeinflußt. Die Abnahme der Mächtigkeit auf dem Mühlenberg-Zug nach $S$ hin erfolgt genau so kontinuierlich wie seitlich davon (s. Abb. 1).

Auch in Hanglange weicht die Mächtigkeit des Sandlösses wenig von der in der Ebene bzw. auf dem Mühlenberg-Zug ab. Am Hang des Mühlenberg-Zuges, bei einem Gefälle von etwa 2,5\%, ist die Mächtigkeit ähnlich wie auf dem Rücken bzw. seitlich davon (s. Abb. 1). Auch unmittelbar westlich Becklingen zeigen sich bei einem Gefälle von 2,2 \% nur geringe Mächtigkeitsschwankungen. Eine Mächtigkeitszunahme am Fuß eines Hanges, die durch Abspülung zu deuten wäre, war lediglich beiderseits des Mühlenberg-Zuges zu beobachten. Sie kommt im Kartenbild (Abb. 1) nur schwach zum Ausdruck und dürfte für ein Sandlößgebiet vergleichsweise gering sein.

*) In den Erläuterungen zu den Blättern 3225 Offen (LANG 1983: Tab. 3 u. 4) und 3322 Steimbke (LANG 1990: in Tab. 47) der Geol. Karte von Niedersachsen 1:25000 sind die Analysen der Sandlößproben in Tabellenform aufgeführt.

\section{Petrographische Ausbildung *}

Nach einem Vorschlag von FiedLeR \& ALTERmanN (1964) werden äolische Ablagerungen dann als Sandlöß bezeichnet, wenn sie mindestens $15 \%$ Sand und mindestens $15 \%$ Schluff mit einem Maximum im Mittel- bis Grobschluffbereich enthalten. Äolische Ablagerungen mit weniger als $15 \%$ Sand wären danach als Löß, mit weniger als $15 \%$ Schluff als Flugsand zu bezeichnen. Nach HINZE (in HINZE et al. 1989) enthält der Sandlöß mindestens $20 \%$ Sand, besteht überwiegend aus Grobschluff und enthält weniger als $20 \%$ Ton bis Mittelschluff. Bezeichnend für den Sandlöß ist ferner eine zweigipfelige Kornverteilungskurve; ein Gipfel liegt meist im Grobschluffbereich, der andere, meist wesentlich schwächere, im Mittelsandbereich.

Der Sandlöß im Raum Bergen ist ausgesprochen schluffreich und sandarm. In einigen Proben steigt der Schluffgehalt auf über $80 \%$ an, sodaß auch ein 2. Gipfel nicht mehr erkennbar ist (Abb. 3). Den Sandlöß im Profil B2/204 würde man, wenn man ihn isoliert ohne Kenntnis des Fundortes betrachtete, als reinen Löß ansprechen, zumal der Tonanteil teilweise auf fast $15 \%$ ansteigt und der Sandgehalt bei der überwiegenden Zahl der Proben dieses Profils unter $15 \%$ bleibt.

Der Sandlöß ist, auch in den mächtigsten Profilen, durchweg kalkfrei. Die Grenze zu seinem Liegenden, hier meist glazifluviatile Sande des Drenthe-Stadiums der Saale-Kaltzeit, ist in der Regel scharf und meist durch eine Steinsohle markiert.

STOLLer (1915a, 1915 b) unterteilte den „Flottsand” auf den Blättern 3126 Hermannsburg und 3226 Sülze der geol. Karte $1: 25000$ in einen maximal $1 \mathrm{~m}$ mächtigen „Geschiebeflottsand" und einen etwa $1 \mathrm{~m}$ mächtigen "gerölle- und geschiebefreien Flottsand". Eine Deutung dieses Befundes gab er nicht.

Auch nach den eigenen Beobachtungen ist der Sandlöß überall dort, wo er nicht mächtiger als $0,7-0,9 \mathrm{~m}$ wird, von einzelnen Geröllen, kleinen Geschieben und groben Sandkörnern durchsetzt, zeigt keinerlei Schichtung und vielfach Wühlspuren. Er sieht weitgehend homogen aus. Geht die Mächtigkeit über dieses Maß hinaus, ist der Sandlöß deutlich zweigeteilt in einen oberen, homogenen Teil, jedoch ohne Gerölle oder Geschiebe, und einen unteren, sandstreifigen Teil, mit einer Wechsellagerung von relativ reinen Schlufflagen mit Sand- bzw. sandreichen Lagen. Diese Streifigkeit wird noch durch unterschiedliche Farben verdeutlicht. Die sandigen bzw. Sandlagen sind meist brauner als die Schlufflagen, möglicherweise durch eingeschwemmten Ton. Die einzelnen Lagen messen meist weniger als $1 \mathrm{~cm}$, ihr Abstand von einander etwa $1-3 \mathrm{~cm}$. 
Die Gerölle- und Geschiebeführung des Sandlösses („Geschiebeflottsand”) ist damit zu erklären, daß bodenwühlende Tiere, die Wurzeln umstürzender Bäume (Bioturbation) und vielleicht auch die Kryoturbation den Sandlöß, wenn er nicht mächtiger als 0,7-0,9 m wurde, mit Material aus dem Liegenden vermischt haben (DEWERS 1931/32). War der Sandlöß mächtiger, so haben Bodenwühler, die Wurzeln umstürzender Bäume und die sommerliche Auftautiefe des sonst tiefgeforenen Bodens das Liegende nicht mehr erreicht. So blieb auch die Schichtung im unteren Teil der mächtigen Sandlöß-Profile erhalten. Daraus ist auch zu folgern (s. auch DEWERS 1931/32 und ROESCHMANN 1963, 1968), daß der Sandlöß ursprünglich durchweg geschichtet war. Diese Schichtung in Sand- bzw. sandreiche Lagen und relativ saubere Schlufflagen kann einmal das Ergebnis verschiedener Windgeschwindigkeiten und der davon abhängigen Transportarten sein. Der Sand wird vornehmlich als „Bodenfracht” bewegt, der Schluff als „Suspensionsfracht" (GLaTTHAaR1981). Jedoch kann die Sandstreifigkeit auch auf wechselnde Windrichtungen hinweisen (s. Kap. 5).

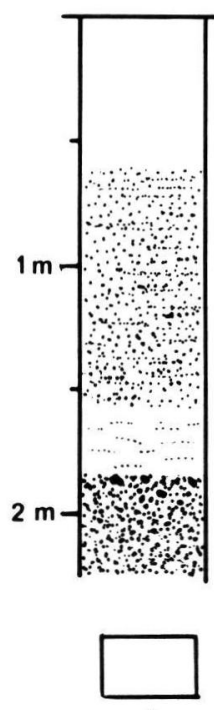

a
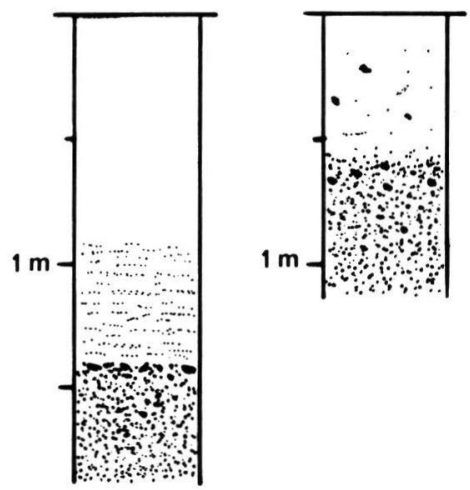

Abb. 2: Die petrographische Ausbildung von Sandlöß in seiner Abhängigkeit von der Mächtigkeit und der Lage innerhalb des Bergener Sandlößgebietes.

links: Sandlößprofil im nördlichen Randgebiet, nahe der Grenze zum Flugsand, darin Flugsandlage mit Schluffbändern

Mitte: Homogener, entschichteter Sandlöß über gebändertem Sandlöß mit Sandstreifen und Sandschlieren, aus dem Gebiet 2-3 km nördlich Bergen

rechts: Geringmächtiger, entschichteter Sandlöß mit kleinen Geschieben bzw. Geröllen und etwas Grobsand, aus dem Raum südöstlich von Bergen
Abb. 2 zeigt in Säulenprofilen Mächtigkeit und Untergliederung von Sandlößprofilen aus dem Untersuchungsgebiet. Das Profil A zeigt einen Sandlöß mit Sandlagen vom äußersten Nordrand des Untersuchungsgebietes, nahe der Grenze zum Flugsand, das Profil B mächtigen Sandlöß, untergliedert in oberen, homogenen und unteren streifigen Sandlöß, das Profil C geringmächtigen, mit Geröllen bzw. Geschieben durchsetzten Sandlöß.

An begünstigten Standorten, wo die Durchlüftung des Bodens besonders gut ist, wie $z$. B. auf dem Mühlenberg-Zug, scheinen bodenwühlende Tiere und auch Pflanzenwurzeln den ursprünglich geschichteten Sandlöß stellenweise auch über die übliche Tiefe hinaus entschichtet zu haben.

Sechs Sandlößprofile, etwa gleichmäßig über das Bergener Sandlößgebiet verteilt, wurden auf ihre Korngrößenzusammensetzung untersucht. Die Lage der untersuchten Profile ist auf Abb. 1 angegeben. Die Proben wurden in gleichmäßigen Abständen über die einzelnen Profile entnommen, und zwar mit einem Stechbohrgerät, einem seitlich geschlitzten, unten angeschärften Rohr von etwa $2 \mathrm{~cm}$ Durchmesser, das mit einem Motorhammer in den Boden getrieben wurde. Jede Probe ist etwa $5 \mathrm{~cm}$ lang; der Abstand vom unteren Ende einer Probe bis zum oberen Ende der nächstfolgenden Probe beträgt $5 \mathrm{~cm}$.

Die Proben wurden 1/100 normaler Ammoniaklösung aufgeschlämmt, anschließend $2 \mathrm{mal} 2$ Minuten mit Ultraschall behandelt. Der Anteil $>20 \mu$ wurde naß abgesiebt, die Fraktion $>63 \mu$ trocken gesiebt. Das verbliebene Material $<20 \mu$ wurde pipettiert. Insgesamt wurden aus 6 Profilen 84 Proben auf ihre Korngrößenteilung hin untersucht.

Es besteht ein deutlicher Zusammenhang zwischen der Korngrößenverteilung und der Mächtigkeit des Profils, aus dem diese Proben stammen, die wiederum, wie in Kap. 3 erläutert, eng an die Lage innerhalb des Bergener Sandlößgebietes gebunden ist. Die folgende Aufstellung zeigt diese Beziehung:

Profil

durchschnittl.

Schluffgehalt

Sandlößmächtigüber das Profil

Bergen B 3/204

Bergen A 2/235

$77,48 \%$

1,7

Bergen B 3/92

$54,27 \%$

3,08

$49,02 \%$

0,9

Bergen B $4 / 66$

Offen A 4/139

$46,3 \%$

0,6

$36,06 \%$

1,0

Offen A 2/109 
Das auf dem Mühlenberg-Zug gelegene Profil B3/204 mit einem extrem hohen Schluffgehalt und einem Tongehalt zwischen $6,3 \%$ und $14,9 \%$ besteht bis $0,7 \mathrm{~m}$ Tiefe aus homogenem, bis $1,7 \mathrm{~m}$ aus sandstreifigem bzw. sandschlierigem Sandlöß, die Profile Bergen A 2/235 und B $3 / 92$ bis 0,95 bzw. $0,6 \mathrm{~m}$ aus homogenem, darunter aus sandstreifigem Sandlöß. Die Profile Bergen B4/66 und Offen A4/139 und A 2/109 zeigen nur homogenen Sandlöß mit einzelnen Geröllen und Geschieben.

Die Abb. 3 zeigt das Korngrößenspektrum aller Proben dieser Profile. Alle Summenkurven der Proben des betreffenden Profils liegen in dem schraffierten Feld bzw. Band. Bis auf die Summenkurven der Proben aus dem Profil B 3/204, das durch den sehr hohen Schluffgehalt auffällt und eher ein Löß ist, zeigen die Summenkurven aller Profile eine deutliche Zweigipfeligkeit. Ferner zeigt sich, mit leichten Abweichungen, daß die Summenkurven bzw. das sie darstellende schraffierte Band um so flacher verlaufen, je geringmächtiger das Profil ist. Der Feinheitsgrad des Sandlösses ist also, mit geringer Variationsbreite, abhängig von der Profilmächtigkeit. Je mächtiger der Sandlöß, um so feiner das Material.

Das Profil A2/235 (Abb. 3) besteht bis $90 \mathrm{~cm}$ Tiefe aus homogenem, darunter bis 3,08 $\mathrm{m}$ Tiefe aus streifigem Sandlöß. Die Sand- bzw. sandreichen Streifen werden meist nur bis etwa $1 \mathrm{~cm}$ mächtig, der Abstand zwischen den Streifen beträgt in der Regel $1-3 \mathrm{~cm}$. Eine Probe über $5 \mathrm{~cm}$ erfaßt also sandarme und sandreiche Streifen. Das waagerecht schraffierte Band in der Abbildung zeigt den Bereich, innerhalb dessen die Summenkurven aller 26 Proben dieses Profils liegen. Das senkrecht schraffierte Feld in dieser Abbildung umfaßt die Summenkurven aller Proben aus dem oberen, homogenen Sandlöß dieses Profils, bis 0,95 m Tiefe. Diese Darstellung macht deutlich, daß sandarme und sandreiche Streifen im Sandlöß zusammengenomen eine sehr ähnliche Korngrößenverteilung zeigen wie ein im selben Profil darüberliegender homogener Sandlöß.

Das bestätigen auch die folgenden Zahlen: Die Proben aus dem oberen, homogenen Sandlöß dieses Profils haben einen durchschnittlichen Schluffgehalt von $56,2 \%$, die Proben aus dem unteren, streifigen Sandlöß einen durchschnittlichen Schluffgehalt von $53,57 \%$. Auch das Ausgangsmaterial für den oberen, homogenen Sandlöß dürfte also ein sandstreifiger Sandlöß gewesen sein.

Stärkere reine Sandlagen fanden sich nur in Sandlößprofilen unmittelbar am Nordrand des Verbreitungsgebietes, wo sie $20-30 \mathrm{~cm}$ mächtig werden. Diese Profile leiten zu Flugsandprofilen über, in denen entweder eingelagert oder an der Basis $30-40 \mathrm{~cm}$ starke
Schlufflagen auftreten. Diese sandige Randfazies des Sandlösses bzw. Übergangsfazies zum Flugsand ist lediglich auf einen schmalen Streifen beschränkt.

Daß die sandigen bzw. Sandlagen im unteren Teil des Sandlösses nicht das Ergebnis einer sekundären Umlagerung sind, zeigt der Aufbau der Profile auf und am Hang des Mühlenberg-Zuges. Die Profile auf dem Mühlenberg-Zug, die besonders um den Proben-Entnahmepunkt B3/204 (vergl. Abb. 1) relativ mächtig sind, zeigen in ihrem unteren Teil genau so eine Sandstreifigkeit wie die Profile am Hang, soweit sie eine entsprechende Mächtigkeit haben. Wäre eine postsedimentäre Umlagerung an der Sandstreifung schuld, sollte sie in den Profilen auf dem Rücken fehlen.

Der relativ hohe Sandgehalt in den geringmächtigen Profilen mit durchgehend homogenem Sandlöß läuft parallel mit einer immer wieder beobachteten Geschiebe- bzw. Geröllführung. Es ist davon auszugehen, daß sowohl Geschiebe bzw. Gerölle als auch Sand durch Bioturbation und Kryoturbation aus dem Liegenden nach Ablagerung des Sandlösses zugemischt wurden.

Auf bzw. am Mühlenberg-Zug und in seiner nächsten Umgebung fällt auf, wie wenig sich Mächtigkeit und Aufbau der Sandlöß-Profile auf dem Rücken von denen am Hang des Rückens unterscheiden (vergl. Abb. 2), zumal der Sandlöß als erionsgefährdet gilt.

Der Sandlöß im Profil B 3/204 (auf dem MühlenbergZug) hat einen hohen Anteil an feinen Bestandteilen (s. Abb. 3). Der Tongehalt steigt bis auf nahezu $15 \%$ an, der Anteil an Sand bleibt meist unter $12 \%$. Es ist zu vermuten, daß der relativ hohe Tongehalt bei gleichzeitig geringem Sandgehalt dem Sandlöß eine unübliche hohe Stabilität verleiht und verhindert, daß er trotz erheblichen Gefälles $(2,5-3 \%)$ stärker erodiert wird.

\section{Die Windrichtung zur Zeit der Sandlöß-Akkumulation}

Die Tatsache, daß der Sandlöß in relativ gleichbleibender Mächtigkeit über Ebenen und Rücken hinwegzieht und auch flache Depressionen auskleidet, darf als weiterer Beweis für seine äolische Entstehung gewertet werden.

Das Bergener Sandlößgebiet liegt östlich des Endmoränen-Hochgebietes um den Falken-Berg (,Falkenberg-Endmoräne"), das das Verbreitungsgebiet des Sandlösses um maximal $80 \mathrm{~m}$ überragt. Nur im Gebiet westlich von Wardböhmen und Becklingen reicht der Sandlöß in das Hochgebiet hinein, scheint 

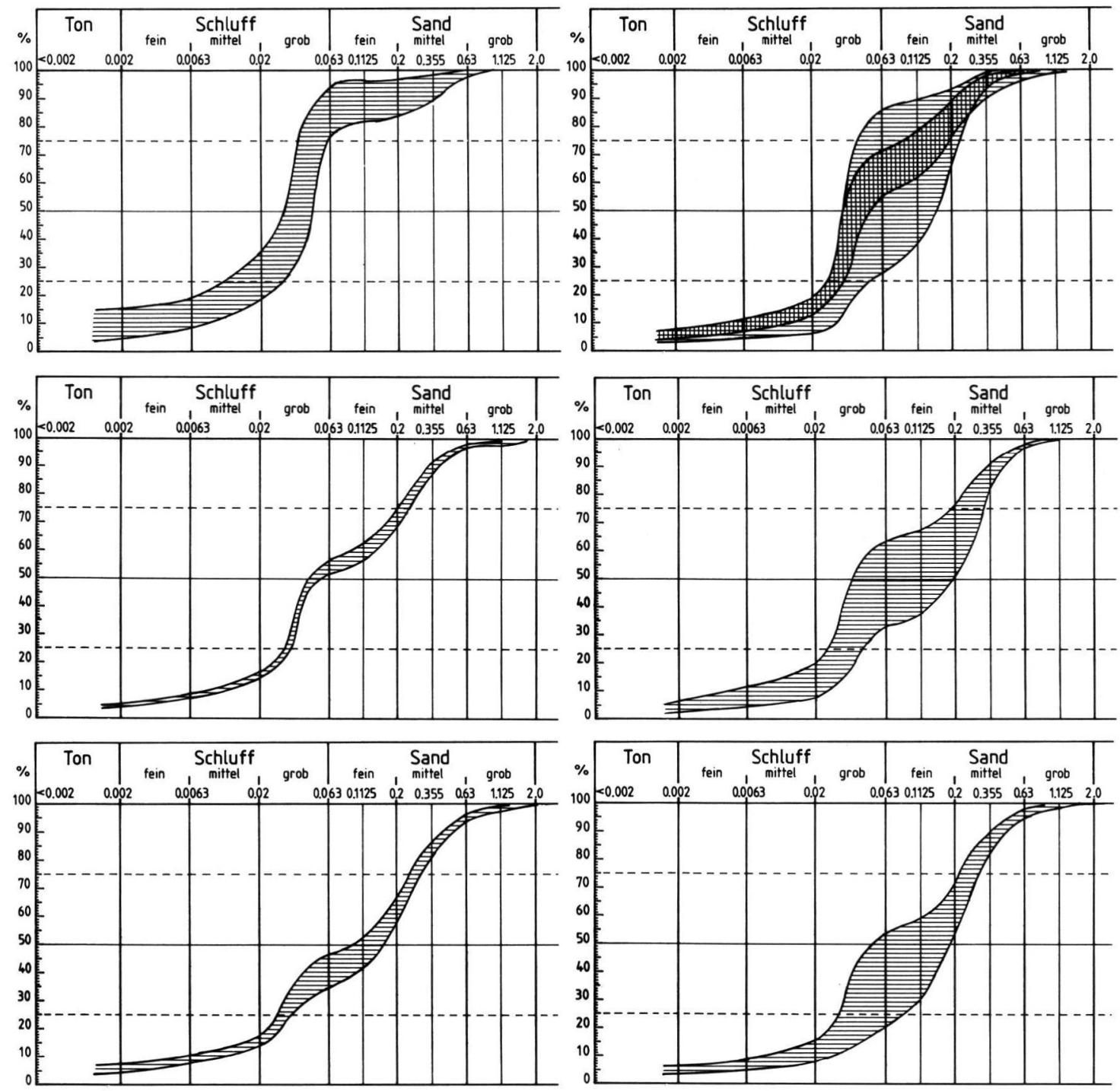

Abb. 3: Die Korngrößenverteilung im Sandlöß, dargestellt in Summenkurven.

Alle Kurven aus dem jeweiligen Profil liegen innerhalb des schraffierten Feldes.

Die Probenentnahmepunkte sind auf Abb. 1 angegeben.

Oben links: Profil Bergen B3/204. 0-0,7 m homogener Sandlöß, bis 1,7 m Sandlöß mit Sandstreifen und Sandschlieren. rechts: Profil Bergen A 2/235. Senkrecht schraffiert: Proben aus 0-0,95 m Tiefe, homogener Sandlöß. -

Waagerecht schraffiert: Proben aus 1,0-3,08 m Tiefe, Sandlöß, geschichtet, mit Sandstreifen und Sandschlieren.

Mitte links: Profil Bergen B 2/92. 0-0,6 m homogener Sandlöß, 0,6-0,9 m Sandlöß mit Sandstreifen und Sandschlieren. rechts: Profil Bergen B4/66. Homogener, mit einzelnen Geschieben bzw. Geröllen und etwas Grobsand durchsetzter

Sandlöß. Liegendes: Mittel- und Feinsand, schluffig.

Unten links: Profil Offen A4/139. Sandlöß wie in Profil B4/66.

Liegendes: Feinsand, mittelsandig.

rechts: Profil Offen A2/109. Sandlöß wie in Profil B4/66.

Liegendes: Fein- bis Mittelsand, leicht schluffig. 
hier allerdings bevorzugt an eine weite, flache Schüssel mit einigen Appendices gebunden zu sein, die vom Hakenberg ( $+143,3 \mathrm{~m}$ üb. $\mathrm{NN}$ ) und einigen Rücken südlich davon mit Höhen um $130 \mathrm{~m}$ üb. NN nach $\mathrm{W}$ begrenzt wird. Davon abgesehen sind das Hochgebiet und sein westliches Vorland frei von Sandlöß. Einige kleine Sandlößfelder liegen südlich des Hochgebietes.

Am südlichen Ortsrand von Becklingen setzt der Sandlöß mit einer deutlichen Geländestufe ein und steigt fast sprunghaft auf eine Mächtigkeit von mehr als $2 \mathrm{~m}$ an. Er ist mit wechselnd mächtigen Sandlagen durchsetzt.

Aus dem Raum unmittelbar westlich Becklingen zieht ein Streifen mächtigen Sandlösses über Wardböhmen in WNW-ESE-Richtung über den Mühlenberg-Zug hinweg bis nahe an die östliche Verbreitungsgrenze (s. Abb. 1). Er ist von feinen Sandbändern und stärkeren Sandlagen, die Schluffbänder enthalten können, durchsetzt.

Der Bereich der größten Sandlößmächtigkeiten liegt unmittelbar östlich des zentralen Teiles des Hochgebietes um den Falken-Berg und den Hakenberg mit Höhen über $140 \mathrm{~m}$ üb. NN., also etwa $40-60 \mathrm{~m}$ höher als der Sandlöß bei Warböhmen und Becklingen.

Diese Befunde sprechen genau so wie die Lage der Flugsandfelder am Nordrand des Sandlößgebietes, die Schlufflagen im Flugsand, die starken Sandlagen im Sandlöß unmittelbar am Nordrand des Verbreitungsgebietes und die Mächtigkeitsabnahme des Sandlösses in südlicher und südöstlicher Richtung für vorherrschende Winde aus nördlicher bis nordwestlicher Richtung zur Zeit der Sandlößakkumulation.

Würde man dagegen versuchen, die Windrichtung unter Vernachlässigung der Mächtigkeiten aus dem Feinheitsgrad des Sandlösses abzuleiten (wie SIEBERZ 1988), käme man leicht zu einer entgegengesetzten Windrichtung. Weisen doch die am weitesten im Süden gelegen Profile mit einem durchschnittlichen Schluffgehalt von $36,03 \%$ bzw. 29,35\% den niedrigsten Feinheitsgrad auf.

Als Herkunftsgebiet für das Feinmaterial des Bergener Sandlösses kommen die großen Grundmoränenflächen im Raum Walsrode-Verden - Visselhövede Schneverdingen bis hin zur Wümme-Niederung bzw. den Raum um Rotenburg infrage, die nordwestlich des Sandlöß-Verbreitungsgebietes liegen. Die Verwitterung der drenthestadialen Grundmoräne dürfte genügend Ton und Schluff für den Sandlöß freigemacht haben.
Nördlich und nordöstlich des Bergener Sandlößgebietes liegen große zusammenhängende Sandgebiete. Sie erstrecken sich östlich der Linie Dorfmark - Soltau Schneverdingen - Buchholz bis nahe Uelzen und Bodenteich hin. In ihnen vermuten wir das Haupt-Herkunftsgebiet des Flugsandes. Die Flugsandfelder am Nordrand des Sandlößgebietes, möglicherweise auch die Sandlagen im Sandlöß, scheinen an vorherrschend nördliche Winde gebunden zu sein.

Die in der Literatur angegebenen Windrichtungen zur Zeit der weichselzeitlichen Sandlöß-Akkumulation decken sich recht gut mit den hier abgeleiteten. VIERHUFF (1967) nimmt allgemein für Sandlösse in Niedersachsen westliche bis nordwestliche Winde an, mit starken lokalen Komponenten, ALTERMANN (1968) und MAUDREI (1968) rechnen für den Sandlöß im Fläming mit Winden aus vorherrschend nördlicher bzw. nördlicher und östlicher Richtung, LANG (1974) für den Sandlöß im Raum Wittingen vorherrschend aus Osten bis Ostnordosten. KOTTMEIER \& MEYER (1988) und MEYER \& KOTTMEIER (1989) haben nach Modellrechnungen für das unmittelbare Eisrandgebiet sowie Osteuropa antizyklonale Ost- und Nordostwinde als effektive Winde ermittelt, für Mittel- und Westeuropa Westwinde. SIEBERTZ (1988) führt die Decksedimente auf dem Niederrheinischen Höhenzug, so weit sie im Hochglazial entstanden sind, auf kontinentale Ostwinde mit einer starken Süd-Komponente zurück, und, so weit sie im ausgehenden Hochglazial und im Spätglazial entstanden sind, auf Südwestwinde mit einer untergeordneten Westwindkomponente. Der Widerspruch zu den zuvor angeführten effektiven Windrichtungen könnte durchaus lagebedingt sein.

\section{Vergleiche mit anderen Sandlößgebieten östlich der Weser}

Die in Niedersachsen östlich der Weser gelegenen Sandlößgebiete sind in nordwestlich-südöstlicher Richtung gestreckt (VIERHUFF 1967). Damit deutet sich eine gewisse Parallelität zum Außenrand des weichselzeitlichen Vereisungsgebietes an, worauf schon WORTMANN (1942) hinwies, auch eine gewisse Parallelität zum Außenrand des warthestadialen Vereisungsgebietes ist angedeutet (LANG 1974). Zu einem Vergleich mit dem Sandlößgebiet um Bergen bieten sich das von Steimbke und das von WittingenHankensbüttel an, die dem Autor aus eigenen Kartierarbeiten vertraut sind.

Das Sandlößgebiet von $\mathrm{S}$ t e i m b k e liegt auf einer nur von wenigen Tälern zerschnittenen Hochfläche unmittelbar südlich der Aller-Niederung. Es ist nach N und NW völlig offen und ungeschützt. Mit 
einer Breite von etwa $6 \mathrm{~km}$ erstreckt es sich über $12,5 \mathrm{~km}$ in nordwest-südöstlicher Richtung. Der Sandlöß wird 1,4 m mächtig. Die größten Mächtigkeiten finden sich entlang des Nordrandes des Verbreitungsgebietes und im äußersten Nordwesten, wo sich der Sandlöß mit schluffreichem Flugsand verzahnt bzw. wo ihm Flugsandflächen nordwestlich vorgelagert sind. Sandlößmächtigkeiten von mehr als $1 \mathrm{~m}$ sind an einen etwa $2 \mathrm{~km}$ breiten Streifen am nördlichen Rand des Verbreitungsgebietes gebunden. Nach JORDAN (1980) erreicht der Sandlöß auf dem südlich anschließenden Blatt 3422 Neustadt am Rübenberge allgemein Mächtigkeiten von $0,5-0,7$ $\mathrm{m}$. Die Mächtigkeit nimmt also auch hier, allerdings wesentlich weniger deutlich als im Bergener Sandlößgebiet, von $\mathrm{N}$ bzw. NE nach $\mathrm{S}$ bzw. SSW hin ab.

Von 3 Profilen aus dem Bereich des Blattes 3322 Steimbke liegen Korngrößenanalysen vor; sie sind bei LANG (1990) aufgeführt. Auch hier weist das mächtige Profil den höchsten Schluffgehalt auf. Es liegt zugleich dem N-Rand des Verbreitungsgebietes am nächsten. Mit nach S abnehmender Profilmächtigkeit nimmt der Anteil an Feinmaterial ab bzw. der Sandgehalt zu.

Untersuchtes Profil

$\begin{array}{lll}\text { Steimbke B4/140a } & 60,95 \% & 1,2 \mathrm{~m} \\ \text { Steimbke B2/59a } & 51,51 \% & 0,6 \mathrm{~m} \\ \text { Steimbke B2/46a } & 44,45 \% & 0,6 \mathrm{~m}\end{array}$

Der Sandlöß in den Profilen B2/59a und B 2/46a ist von einzelnen kleinen Geschieben bzw. Geröllen durchsetzt. Das Liegende beider Profile besteht aus Grundmoräne, deren oberste Schichten in einen geschiebeführenden, schluffigen Sand umgewandelt sind, bei Profil B 4/140a aus schwach mittelsandigem Feinsand.

Ein Vergleich mit den von JORDAN (1980) in Form von einzelnen Summenkurven aufgeführten Analysen ist nur bedingt möglich, da hier Gesamtmächtigkeiten nicht aufgeführt sind. Aber auch hier ist es so, daß die Probe mit dem höchsten Schluffgehalt aus dem mächtigsten der beprobten Profile (aus 0,8 m Tiefe) stammt.

Eine Unterteilung in einen homogenen und einen sandstreifigen Sandlöß ist, der allgemein geringen Mächtigkeiten wegen, nur in wenigen Profilen vornehmlich am nordwestlichen Rand des Verbreitungsgebietes nahe der Grenze zu Flugsandfeldern erkennbar; sie ist auf mehr als $0,9 \mathrm{~m}$ mächtige Profile beschränkt.
Das Sandlößgebiet von W it t i n g e n - H a n $\mathrm{k}$ e $\mathrm{n} \mathrm{s} \mathrm{b}$ ü t $\mathrm{t}$ e l (LANG 1969, 1974; DUPHORN 1969) ist mit seiner NW-SE-Erstreckung von etwa $25 \mathrm{~km}$ bei einer maximalen Breite von etwa $8 \mathrm{~km}$ wesentlich größer als das von Steimbke und auch von Bergen, jedoch bei maximalen Sandlößmächtigkeiten von nur 1,1 bis 1,2 m. Entsprechend weniger deutlich sind die Gesetzmäßigkeiten in der Mächtigkeitsentwicklung. Zusammenhängende Flächen mit einer Sandlößmächtigkeit von $0,8 \mathrm{~m}$ und mehr liegen im wesentlichen in einem Streifen zwischen Sprakensehl und Erpensen und dem Raum unmittelbar nördlich Wittingen, folgen also auch dem Nordrand des Verbreitungsgebietes, der auf der Höhe von Wittingen leicht nach S abbiegt. Die größten Mächtigkeiten finden wir im NW-Teil des Verbreitungsgebietes. Flugsand kommt in unmittelbarer Nähe nicht vor.

Korngrößenanalysen haben wir aus diesem Gebiet nicht. Die bisherige Tendenz einer Zunahme des Sandgehaltes mit abnehmender Profilmächtigkeit deutet sich hier bereits im Feldbefund an, ebenso die Unterteilung in oberen, ungeschichteten Sandlöß und unteren, sandstreifigen, geschichteten in den mächtigeren Profilen (LANG 1974).

Im Raum Wittingen - Hankensbüttel überdeckt der Sandlöß ein leicht hügeliges Gelände, das von etwa $115 \mathrm{~m}$ üb. NN im NW auf etwa $90 \mathrm{~m}$ im S und SE abfällt. Es ist nach allen Seiten offen und ungeschützt.

\section{Schriftenverzeichnis}

AltermanN, M. (1968): Quartärgeologische und bodenkundliche Untersuchungen an weichselzeitlichen äolischen Deckschichten im Gebiet Sachsen-Anhalt. - Dissertation Techn. Univ. Dresden.

DEWERS, F. (1931/32): Flottsandgebiete in Nordwestdeutschland, ein Beitrag zum Lößproblem. - Abh. Nat. Ver. Bremen, 28, Sonderh.: 131-204, 6 Abb., 2 Taf.; Bremen.

DupHORN, K. (1969): Kartierbericht Wittingen und Brome Nr. 3230 und 3331. - Ber. Arch. Nieders. Landesamt f. Bodenforschung: $11 \mathrm{~S}$; Hannover. - [Unveröff.]

- (1972): Geologie, in: Der Landkreis Gifhorn. Amtliche Kreisbeschreibungen, 26: 22-30, 1 Abb., 1 Ta.; Bremen-Horn (Dorn-Verlag).

Fiedler, H. J. \& AltermanN, M. (1964): Verbreitung, Entstehung und Eigenschaften von Sandlöß („Flottsand”) im norddeutschen Flachland und angrenzenden Gebieten. - Geologie, 13: 1199-1228, 27 Abb., 1 Tab.; Berlin.

GlatthaAR, D. (1981): Sandlöß bei Damme - Borringhausen. Beiträge zur Glazialmorphologie und zum periglaziären Formenschatz. - Bochumer geograph. Arb., 40: 121, Abb. 17; Paderborn. 
Hinze, C. in: Hinze, C., Jerz, H., Menke, B. \& Staude, H. (1989): Geogenetische Definitionen quartärer Lockergesteine für die Geologische Karte 1:25000 (GK 25). - Geol. Jb., A 112: S. 161; Hannover.

JoRDAN, H. (1980): Geol. Karte Niedersachsen 1:25000, Erl. Blatt 3422 Neustadt am Rübenberge, mit Beiträgen von E. Cosak, E. Dahms, W. EckelmanN, E. Groba, W. IRRLITZ, F. KOCKEL, H. SCHNEEKLOTH \& J. TUXEN. 88 S., 14 Abb., 4 Tab., 4 Kt.; Hannover (Nieders. L.Amt Bodenforsch).

Klausing, C. (1990): Böden, in Lang, H. D.: Erl. Geol. Kt. Niedersachsen 1:25000, Bl. 3125 Bergen. Hannover (Niederr. L.-Amt Bodenforsch.). - [Im Druck].

KotTMeier, C. \& Meyer, H.-H. (1988): Ein einfaches analytisches Modell zur Darstellung der atmosphärischen Zirkulation in Europa im Weichsel-Hochglazial. - Erdkunde, 42: 261-273, 7 Abb., 1 Tab.; Bonn.

LANG, H. D. (1974): Über Verbreitung, Zusammensetzung und Alter des Sandlößes im Raum Wittingen Hankensbüttel. - Z. dt. geol. Ges., 125: 269-276, 3 Abb., 1 Tab.; Hannover.

- (1983): Geol. Kt. Niedersachsen 1:25000, Erl. Bl. 3225 Offen. - 108 S., 8 Abb., 5 Tab., 8 Kt.; Hannover (Nieders. L.-Amt Bodenforsch.).

- (1990): Geol. Kt. Niedersachsen 1:25000, Erl. Blatt 3322 Steimbke. - 8 Abb., 9 Tab., 1 Taf., 7 Kt.; Hannover (Nieders. L.-Amt Bodenforsch.). - [Im Druck].

MAUDREI, F. (1968): Geomorphologische, stratigraphische und paläogeographische Untersuchungen im Pleistozän des Niederen Fläming. - Diss. Univ. Berlin.
MeYeR, H.-H. \& KotTMeIeR, C. (1989): Die atmosphärische Zirkulation in Europa im Hochglazial der WeichselEiszeit - abgeleitet von Paläowind-Indikatoren und Modellsimulationen. - Eiszeitalter u. Gegenwart, 39: $10-18,4 \mathrm{Abb}$; Hannover.

RoEschmanN, G. (1963): Zur Entstehungsgeschichte von Parabraunerden und Pseudogleyen aus Sandlöß südlich von Bremen. - N. Jb. Geol. Paläont. Abh., 117 (Festband Lotze): 286-302, 1 Tab., 1 Taf.; Stuttgart.

- (1968): Besonderheiten der Ton- und Eisenverlagerung in Parabraunerden aus Sandlöß südwestlich von Bremen. — Mitt. Dt. Bodenkundl. Ges., 8: 277-282, 1 Abb.; Göttingen

SieberTZ, H. (1988): Die Decksedimente auf den Niederrheinischen Höhenzug in ihrer Beziehung zu den Luftdruck- und Windverhältnissen während der WeichselKaltzeit in Nordwestdeutschland. - Natur am Niederrhein, 3,1: 1-12, 8 Abb., 3 Tab.; Krefeld.

Stoller, J. (1915a): Erl. Geol. Kt. 1 : 25000 von Preußen, Bl. 3126 Hermannsburg: 61 S., 1 Kt., 10 Tab.; Berlin (Preuß. Geol. L.-Anst.).

- (1915b): Erl. Geol. Kt. von Preußen 1:25000, Bl. 3226 Sülze. - 61 S., 1 Kt., 4 Abb., 9 Tab.; Berlin (Preuß. Geol. L.-Anst.).

VIERHUFF, H. (1967): Untersuchungen zur Stratigraphie und Genese der Sandlößvorkommen in Niedersachsen. - Mitt. Geol. Inst. TH Hannover, 5: 99 S., 35 Abb.; Hannover.

Wortmann, H. (1942): Bemerkungen zu einer Karte der Lößverbreitung in Niedersachsen. - Arch. Landes- u. Volkskde. von Niedersachsen, 12: 192-202; Oldenburg.

Manuskript eingegangen am 15. 12. 1989. 